\title{
P01-193 - DEVELOPMENTAL COMORBIDITY ASSESSMENT IN CHILDHOOD ADHD
}

\section{P. Dineen ${ }^{1}$, M. Fitzgerald ${ }^{2}$}

${ }^{1}$ Child Psychiatry, Linn Dara Child \& Adolescent Mental Health Services, ${ }^{2}$ Child Psychiatry, Trinity College Dublin, Dublin, Ireland

Introduction: Childhood ADHD typically presents with comorbidites. Assessment tools for developmental screening vary greatly in length and complexitity, and parental preference has an impact on the usefulness of clinical tools in routine practice.

Objectives: To determine among a group of children which ones may benefit from occupational therapy, speech and language therapy and educational progress re-assessment as part of routine clinical assessment for ADHD with the intention to improve service delivery.

Aims: Children were referred on a routine basis to a community clinic for ADHD assessment. SNAP-IV, FTF and BRIEF rating scales were administered. The FTF was used as a parent-rated screen for general development; it is a 200-item questionairre and includes sections on motor skills, speech and language skills and variety of other areas.

Methods: A review of freely available developmental questionaires was conducted. The FTF was introduced on a trial basis as a balance between the typical shorter scale of the SDQ and longer time needed for the DAWBA.

Results: The parents of 25 children completed the FTF as part of routine clinical assessment for ADHD. Along with identifying groups of children who could benefit from further allied-healthcare assessment, the majority of parents had no difficulty with the length, although minority did report difficulty with understanding the language. Diagnoses were confirmed using SNAP-IV and clinical interview and assessment. Executive function scores were compared to FTF executive function screening scores.

Conclusions: Routine screening of developmental comorbidities may help with the development of patient -related outcome measures. 